\title{
REFERENCES
}

1. M. Altman, On the approximate solution of non-linear functional equations, Bull. Acad. Polon. Sci. Cl. III vol. 5 (1957) pp. 457-460.

2. Concerning approximate solutions of non-linear functional equations, Bull. Acad. Polon. Sci. Cl. III vol. 5 (1957) pp. 461-465.

3. - On the approximate solutions of non-linear functional equations in Banach spaces, Bull. Acad. Polon. Sci. Ser. Math. vol. 6 (1958) pp. 19-24.

4. L. V. Kantorovich, Some further applications of Newton's method to functional equations (in Russian), Vestnik Leningrad. Univ. Ser. Mat. vol. 2 (1957) pp. 68-103.

California Institute of Techology and Academy of Science, Warsaw

\section{AN UNCOUNTABLE SET OF INCOMPARABLE DEGREES}

\section{J. R. SHOENFIELD}

The purpose of this note is to prove the following: ${ }^{1}$

THEOREM. There is an uncountable set of pairwise incomparable degrees of recursive unsolvability.

By Zorn's lemma, there is a maximal set of pairwise incomparable degrees of recursive unsolvability different from 0 ; we must show that this set is not countable. Hence our theorem follows from:

LEMmA. If $a_{0}, a_{1}, \cdots$ is a sequence of degrees different from 0 , then there is a degree $b$ which is incomparable with each $a_{n}$.

Proof. ${ }^{2}$ Let $\alpha_{n}$ be a function of degree $a_{n}$; we shall construct a function $\beta$ of degree $b$. As in [1], $\beta$ is constructed by defining inductively a function $\kappa$ such that $\kappa(a)=\bar{\beta}(\nu(a))$ with $\nu(a)=\ln (\kappa(a)) ; \kappa$ and $\nu$ must satisfy the conditions that $\kappa(a)$ is a sequence number, $\kappa(a+1)$ extends $\kappa(a)$, and $\nu(a+1)>\nu(a)$. We then have $\beta(a)=(\kappa(a+1))_{a}$ -1 .

Let $\kappa(0)=1$. To define $\kappa(a+1)$, let $n=(a)_{1}$ and $e=(a)_{2}$. If $a$ is even, set

$$
\kappa(a+1)=\kappa(a) \cdot p_{\nu(a)} \exp \left(\{e\} \alpha_{n}(\nu(a))+2\right)
$$

if $\{e\} \alpha_{n}(\nu(a))$ is defined, and $\kappa(a+1)=\kappa(a) \cdot p_{\boldsymbol{r}(a)}$ otherwise. Then clearly $\beta \neq\{e\} \alpha_{n}$ for any function $\beta$ such that $\beta(\nu(a+1))=\kappa(a+1)$.

Received by the editors April 29, 1959.

1 The problem solved in this paper was suggested to the author by C. Spector.

2 We use the notation of [1] in the proof. 
If $a$ is odd, we shall choose $\kappa(a+1)$ so that $\alpha_{n} \neq\{e\}^{\beta}$ for any function $\beta$ such that $\bar{\beta}(\nu(a+1))=\kappa(a+1)$. Since $\alpha_{n}$ is not recursive, there is a $w$ such that $\alpha_{n}(w)$ is not equal to

$$
U\left(\mu y\left[\operatorname{Ext}(y, \kappa(a)) \& T_{1}^{1}(y, e, w)\right]\right) .
$$

If (1) is undefined, i.e., if $(\bar{E} y)\left(\operatorname{Ext}(y, \kappa(a)) \& T_{1}^{1}(y, e, w)\right)$, then $\{e\}^{\beta}(w)$ is necessarily undefined, and we may take $\kappa(a+1)=\kappa(a) \cdot p_{\nu(a)}$. Otherwise, there is a $y$ such that $\operatorname{Ext}(y, \kappa(a)), T_{1}^{1}(y, e, w)$, and $U(y)$ $\neq \alpha_{n}(w)$. We may then take $\kappa(a+1)$ to be any extension of $y$ of length greater than $\nu(a)$.

Since $\beta \neq\{e\}^{\alpha_{n}}$ and $\alpha_{n} \neq\{e\}^{\beta}$ for all $e, \boldsymbol{b}$ is incomparable with $\boldsymbol{a}_{n}$.

\section{ReFERENCE}

1. S. C. Kleene and Emil L. Post, The upper semi-lattice of degrees of recursive unsolvability, Ann. of Math. vol. 59 (1954) pp. 379-407.

Duke University 